\title{
New concepts and results in laser power stabilization
}

\author{
P. Kwee $\cdot$ B. Willke $\cdot$ K. Danzmann
}

Received: 30 June 2010 / Revised version: 24 September 2010

(c) Springer-Verlag 2011

\begin{abstract}
In the recent decade the development of highpower, high-sensitivity photodetectors and low-noise sensing techniques lead to achievable relative power stabilities at the level of $10^{-9} \mathrm{~Hz}^{-1 / 2}$ for frequencies between about $10 \mathrm{~Hz}$ and $10 \mathrm{kHz}$. A new power stabilization concept involving the novel optical ac coupling technique complements these high-sensitivity photodetectors. This technique improves the sensitivity of a photodetector by about one order of magnitude. Furthermore, it beats the quantum limit of traditional power stabilization concepts and opens a whole new range of attainable power stabilities that seemed to have been inaccessible due to technical limitations before. In this article we review various laser power stabilization experiments in the field of ground-based gravitational wave detectors, present the best results and discuss their limits.
\end{abstract}

\section{Introduction}

Laser power stabilization is important for many optics experiments. Often the stabilization is part of a more complex experiment where the power fluctuations of the freerunning laser would mask faint signals or would limit the sensitivity of the experiment. In particular gravitational wave detectors (GWDs) require one of the most demanding

P. Kwee $(\bowtie) \cdot$ B. Willke $\cdot$ K. Danzmann

Max-Planck-Institut für Gravitationsphysik

(Albert-Einstein-Institut), Leibniz Universität Hannover,

Callinstr. 38, 30167 Hannover, Germany

e-mail: patrick.kwee@aei.mpg.de

B. Willke

e-mail: benno.willke@aei.mpg.de

K. Danzmann

e-mail: karsten.danzmann@aei.mpg.de power stabilities of today for the laser beam to be injected into the interferometer [1,2]. The free-running laser power fluctuations of the high-power, highly stable laser systems used [3] would limit the sensitivity of second-generation GWDs [4-7] by several orders of magnitude. Therefore active laser power stabilization is an indispensable part of these instruments.

Laser power fluctuations couple to the gravitational wave readout channel directly in the photo-detection process at the interferometer readout and via radiation pressure. Second-generation GWDs will enhance the laser beam power by optical resonators in the interferometer up to a circulating power of nearly $1 \mathrm{MW}$. This laser beam will be reflected at test masses suspended with multi-stage pendulums and will cause radiation pressure. Differential distance fluctuations between these test masses have to be measured to a precision of about $10^{-19} \mathrm{~m}$ in order to reach the design sensitivity of these instruments. Small fluctuations of the laser power cause radiation pressure fluctuations acting on the test mass which leads to length changes of the kilometer-length baseline interferometer arms. Asymmetries will cause slightly different power levels in the interferometer arms such that power fluctuations cause a differential length change that cannot be distinguished from length changes due to gravitational waves. By this effect, laser power fluctuations couple to the gravitational wave channel and thus an active power stabilization of the laser is essential in the gravitational wave detection frequency band of about $10 \mathrm{~Hz}$ to $10 \mathrm{kHz}$.

A high-sensitivity power sensor, transforming laser power into an electrical signal, is the crucial component in most power stabilization experiments. Hence highly sensitive photodetectors developed for GWDs might become essential components in many optics experiments, such as 
sensors in optical communications, the detection of bright squeezed light, or laser frequency stabilizations.

The sensitivity of a photodetector is limited in principle by technical noise and quantum noise. Quantum noise is a fundamental limit caused by the quantization of the light energy into photons, or the quantization of the photocurrent into electrons after the photo-detection process. This limit can be surpassed only by non-classical states of light, such as squeezed states, which are to our knowledge not used in any power stabilization experiment due to their complex generation and fragility until now.

The single-sided linear spectral density $s_{\mathrm{q}}$ of the relative quantum power noise is

$$
\begin{aligned}
s_{\mathrm{q}} & =\sqrt{\frac{2 \times h c}{P \lambda}} \\
& =1.9 \times 10^{-8} \mathrm{~Hz}^{-1 / 2} \times \sqrt{\frac{1 \mathrm{~mW}}{P}} \times \sqrt{\frac{1064 \mathrm{~nm}}{\lambda}},
\end{aligned}
$$

where $h$ is the Planck constant, $c$ is the speed of light in vacuum, $\lambda$ is the vacuum wavelength, and $P$ is the detected laser power. The quantum power noise is independent of the Fourier frequency and often called shot noise. In case a photodetector is used as power sensor this quantum-noise level can also be calculated using the photocurrent $I$

$s_{\mathrm{q}}=\sqrt{\frac{2 e}{I}}=1.8 \times 10^{-8} \mathrm{~Hz}^{-1 / 2} \times \sqrt{\frac{1 \mathrm{~mA}}{I}}$,

where $e$ is the elementary charge.

In order to improve the quantum-noise-limited sensitivity of a photodetector, the detected laser power $P$ and with it the photocurrent $I$ have to be increased. The high power in turn causes most technical problems, such as photodiode saturation, dynamic range limits of the readout electronics, or thermal effects in the photodiode.

We took two different, complementary approaches in the last few years to successfully increase the effective detected laser power and with it the sensitivity of the photodetector: One approach was scaling the number of photodiodes leading to a high-power high-sensitivity photodiode array. The other approach was using optical resonators to increase the sensitivity of a photodetector without increasing the average photocurrent.

In the following sections the most important power stabilization experiments in the field of GWDs are reviewed including a detailed description of the mentioned photodiode array, and the new stabilization concepts involving a technique we called optical ac coupling is described.

\section{Traditional power stabilization experiments}

In the recent decade several power stabilization experiments, among others [8-17], were performed specifically

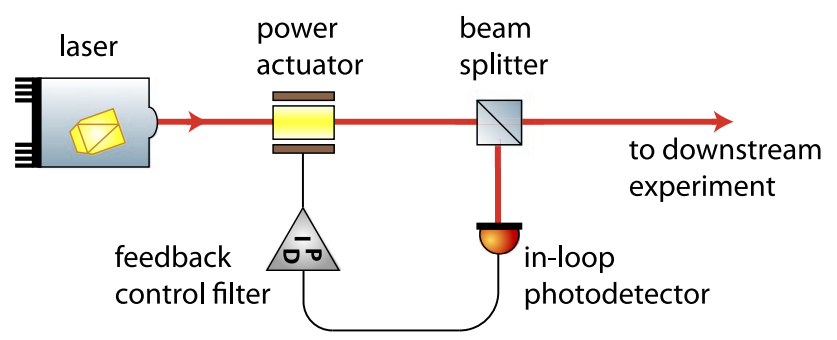

a Traditional power stabilization

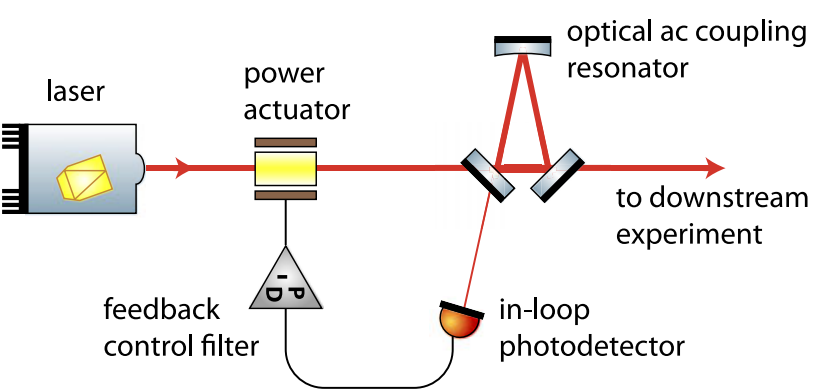

b Power stabilization with optical ac coupling

Fig. 1 Active laser power stabilization schemes. (a) Traditional scheme, commonly used until now, and (b) new scheme involving the optical ac coupling technique

for GWDs. Most stabilizations use photodetectors, feedback control loops, and various actuators to reduce technical laser power noise in a frequency band from about $10 \mathrm{~Hz}$ to $10 \mathrm{kHz}$. The stabilization scheme (Fig. 1a) is very similar in these experiments: The laser beam is split into two parts; one part is detected by a power sensor used for the stabilization (in-loop photodetector) and the other part is used for the actual downstream experiment. The power fluctuations measured by the in-loop detector are suppressed with a feedback control filter and a power actuator. In most power stabilization experiments the downstream experiment contains a second power sensor, called out-of-loop detector, used for an independent measurement of the actually achieved power stability. In order to distinguish this commonly used active power stabilization concept from new concepts it is called traditional power stabilization in this article.

Different continuous-wave, single-frequency lasers at a wavelength of $1064 \mathrm{~nm}$ were used in these experiments with output powers ranging from about $200 \mathrm{~mW}$ to $12 \mathrm{~W}$. Many experiments use the laser pump power to control the output power of the system [9-14]. This is a good choice since pump power fluctuations often cause the laser output power fluctuations in the frequency band of interest. Some experiments $[15,17]$ used external power actuators, such as acousto-optical modulators (AOMs) or electro-optic amplitude modulators (EOAMs) as they have reduced crosscouplings to laser beam parameters other than the beam power. 


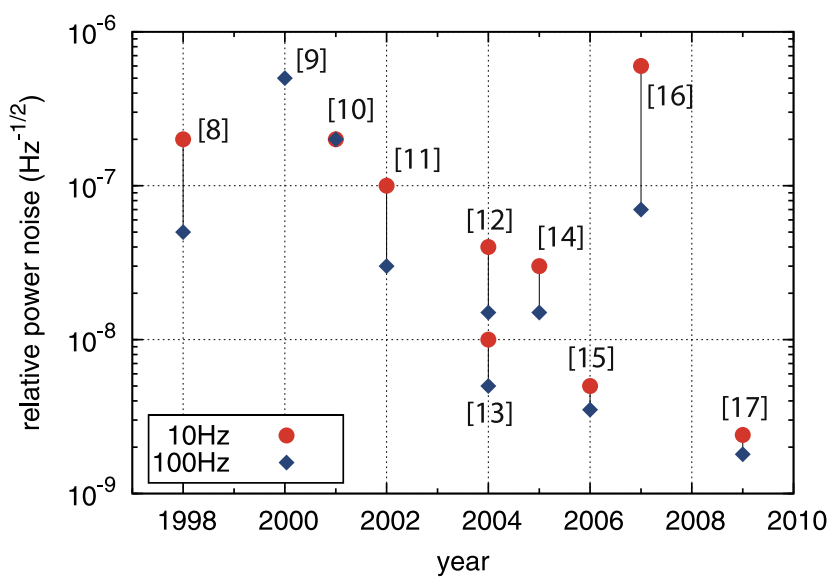

Fig. 2 Out-of-loop measured residual power noise at $10 \mathrm{~Hz}$ and $100 \mathrm{~Hz}$ of several traditional power stabilization experiments from 1998 to 2009

However in most experiments the performance achieved was not limited by the actuators or the feedback control electronics but by noise sources coupling into the power sensor of the control loop. Thus in many experiments significant, unexplained differences showed up in the noise levels measured by the in-loop and out-of-loop photodetector. In the ideal case the in-loop and out-of-loop detector are solely limited by quantum noise [18]. However the power noise measured out-of-loop is often higher than the expected quantum-noise level, especially at low frequencies of a few tens of hertz. A low noise-level measured with the in-loop detector verifies only that the feedback control loop is performing well. In most cases it is impossible to deduce the actually achieved power stability in the downstream experiment using the in-loop photodetector signal since laser power noise cannot be distinguished from sensor noise of the in-loop detector. Therefore an independent, out-of-loop measurement is very important.

Many different technical noise sources were identified that in principle can cause this discrepancy between inloop and out-of-loop noise, such as photodiode internal low-frequency noise, resistor current noise, beam pointing in combination with spatial inhomogeneous photodiodes, dust particles passing the beam, temperature fluctuations, photodiode bias voltage fluctuations, scattered light, electronic grounding noise, or polarization fluctuations [13-15, 17, 19].

The out-of-loop residual power noise achieved in these selected experiments [8-17] is shown in Fig. 2. The best power stability so far, to our knowledge, in the gravitational wave frequency band was achieved with our high-sensitivity photodiode array [17]. This experiment is described in more detail in the following subsection.

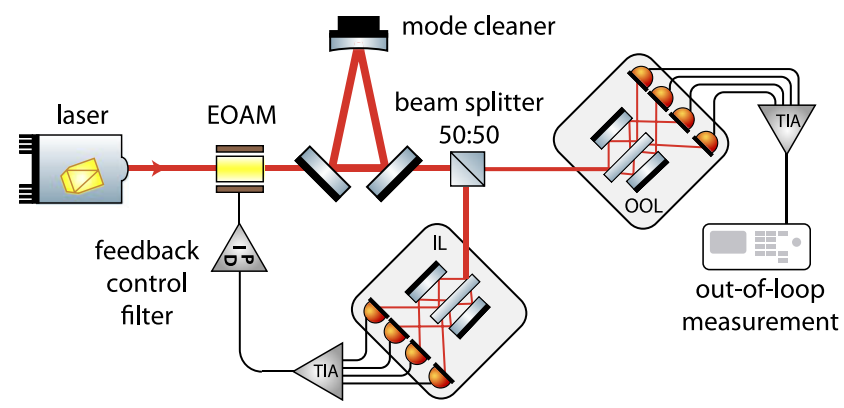

Fig. 3 Simplified setup of the photodiode array power stabilization experiment. The power fluctuations of the laser are measured with the in-loop photodetector IL and are compensated by the feedback control loop using the electro-optic amplitude modulator EOAM. The photodetector OOL is used for an independent measurement of the achieved power stability

\subsection{High-sensitivity photodiode array}

In this experiment we used a Nd:YAG nonplanar ring oscillator (NPRO) [20] as laser with an output power of about $2 \mathrm{~W}$ and an EOAM as the laser power actuator (Fig. 3). As already pointed out the most critical component in a power stabilization at these frequencies is a low-noise power sensor. We used a high-sensitivity photodiode array as power sensor. This power detector was specifically developed for the second-generation GWD Advanced LIGO (Laser Interferometer Gravitational-Wave Observatory) [4] and stands out due to its vacuum compatibility, a low-noise readout electronics, and a low-scattering optical design.

The photodiode array and an upstream ring resonator were placed in a pressure-tight tank for acoustic shielding which was filled with dust filtered air. The ring resonator was used as mode cleaner and reduced beam pointing fluctuations. The beam $(\approx 500 \mathrm{~mW})$ was split with $50: 50$ beam splitters into eight partial beams of nearly equal power (57-65 $\mathrm{mW}$ ) using multiple reflections. The photodiode array consisted of eight InGaAs photodiodes with an active diameter of $2 \mathrm{~mm}$. The electronics for the photodiodes were placed outside of the tank. Each photodiode was connected to a low-noise transimpedance amplifier with a low currentnoise $200 \Omega$ resistor. At the operation point each photodiode detected a photocurrent of about $50 \mathrm{~mA}$. Four signals were added and used to stabilize the laser power (in-loop), and the remaining four signals were added and used to verify the power stability (out-of-loop). For the stabilization the in-loop signal was subtracted from a lowpass-filtered voltage reference, amplified in analog servo electronics, and fed back to the EOAM. This dc-coupled feedback control loop had a bandwidth of about $80 \mathrm{kHz}$ with a loop gain of more than $68 \mathrm{~dB}$ for frequencies below $1 \mathrm{kHz}$.

We found a free-running relative power noise downstream of the mode cleaner of $10^{-7}-10^{-6} \mathrm{~Hz}^{-1 / 2}$ in the frequency band from $1 \mathrm{~Hz}$ to $1 \mathrm{kHz}$. With closed feedback 


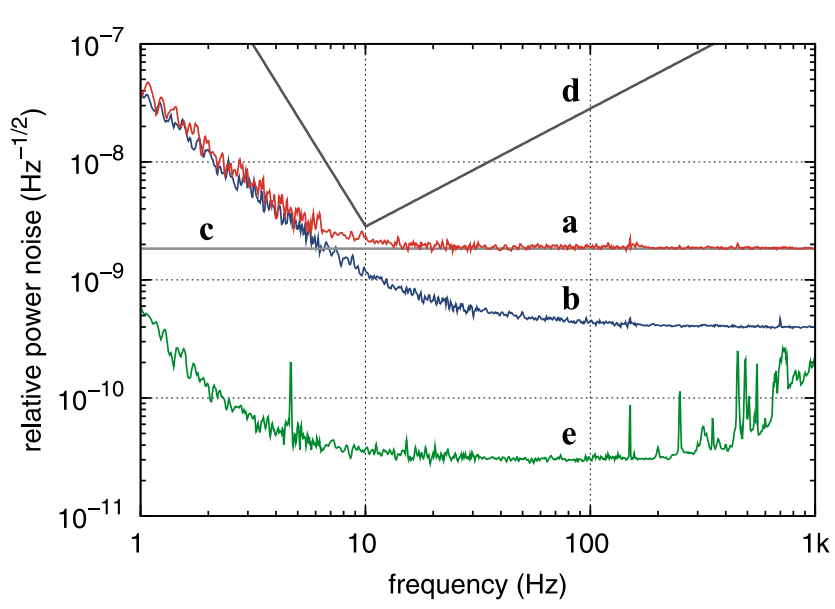

Fig. 4 Power noise measured out-of-loop (a) with the photodiode array. For low frequencies, the measurement was limited by electronic noise (b) and for frequencies above $7 \mathrm{~Hz}$ by quantum noise (c) of the in-loop (200 mA photocurrent) and out-of-loop detector (189 mA photocurrent). The in-loop measured power noise (e) is far below these limiting noise sources. The Advanced LIGO power-noise requirement (d), shifted by $3 \mathrm{~dB}$ in the plot to account for the noise of the out-of-loop detector, was met in the whole frequency band

loop the out-of-loop measured power noise (Fig. 4) was at the expected level defined by the uncorrelated sum of the quantum noise and electronic noise of both, the in-loop and out-of-loop detectors in the whole frequency band. For frequencies up to $7 \mathrm{~Hz}$ the measured noise was dominated by the electronic noise of the in-loop and out-of-loop detector and for higher frequencies by quantum noise at a level of $1.8 \times 10^{-9} \mathrm{~Hz}^{-1 / 2}$. At $10 \mathrm{~Hz}$ we measured a relative power noise of $2.4 \times 10^{-9} \mathrm{~Hz}^{-1 / 2}$ at the out-of-loop detector.

Since the in-loop and out-of-loop detectors were built equally we assumed that the noise of in-loop and out-ofloop detector contributed equally to the measurement noise. With this assumption and by subtracting the noise contribution of the out-of-loop detector we deduced a $3 \mathrm{~dB}$ lower relative power noise of the laser beam of $1.7 \times 10^{-9} \mathrm{~Hz}^{-1 / 2}$ at $10 \mathrm{~Hz}$ and $1.3 \times 10^{-9} \mathrm{~Hz}^{-1 / 2}$ at higher frequencies.

In initial experiments the achieved power stability was limited by technical noise sources, such as lateral beam jitter. However the coupling factor between beam jitter and the out-of-loop signal has been kept small by appropriate alignment of the photodiodes to the incident beam for the final experiment. Furthermore it was important to perform the experiment in filtered air. With unfiltered laboratory air (particle count of about $8500 \mathrm{~m}^{-3} \approx 250 \mathrm{ft}^{-3}$, particle size $\geq 0.3 \mu \mathrm{m}$ ) it was impossible to take a stationary measurement due to signal glitches caused by particles.

To our knowledge this is the first experiment in this power stability range for which the limiting noise sources were fully identified, which was closer than $1 \mathrm{~dB}$ to the quantumnoise limit for frequencies above $15 \mathrm{~Hz}$, and which fulfilled the required but hitherto unattained power stability of $2 \times 10^{-9} \mathrm{~Hz}^{-1 / 2}$ at $10 \mathrm{~Hz}$ for Advanced LIGO. Further details about this experiment can be found in [17, 21].

\section{New stabilization concepts}

To achieve higher power stabilities in the future the sensitivities of the power sensors have to be improved by reducing the relevant noise sources. Technical noise sources, which are often limiting at low frequencies of a few tens of hertz, seem to be controllable by carefully designed experiments (see Sect. 2). In contrast quantum noise is a fundamental noise source. In the traditional detection technique, commonly used until now, the relative quantum noise can be reduced only by increasing the detected laser power. However, the traditional power stabilization experiments (see Fig. 2) and our own experiences indicate that the power limit of photodiodes commonly used is reached. This limit seems to be roughly between about $20 \mathrm{~mA}$ and $50 \mathrm{~mA}$ if a quantumnoise-limited performance at low frequencies around $10 \mathrm{~Hz}$ is required. Thus the sensitivity of this traditional detection technique can be improved only by further scaling the number of photodiodes. The technical effort is roughly proportional to the number of photodiodes or the beam power detected, whereas the sensitivity increases only with the square root of the power detected. Thus today a sensitivity for relative power noise at, e.g., the $10^{-10} \mathrm{~Hz}^{-1 / 2}$ level seems to be out of reach or only achievable with extreme effort when using traditional detection techniques.

The high beam power that needs to be detected causes the most severe technical problems. In the traditional detection technique the light field carrier that contains almost the complete beam power has to be detected, although only the sidebands contain the information about the power fluctuations to be measured. In contrast, with advanced techniques involving optical resonators it is possible to reduce the average power on the photodetector while keeping the same quantum-noise-limited sensitivity of power fluctuations. We investigated different power stabilization concepts [21, 22]. The concept using a detection technique called optical ac coupling [23] was the most promising, especially at the high laser power levels that will be used in future interferometric GWDs. With this technique, which is explained in more detail in the following subsection, we were able to increase the sensitivity of a photodetector by about one order of magnitude. To achieve the same effect in a traditional detection setup, one would have to increase the detected laser power by a factor of 100 .

\subsection{Optical ac coupling}

The optical ac coupling technique is a high-sensitivity detection method for power fluctuations, and it is based on photodetection in reflection of an optical resonator with a specific 


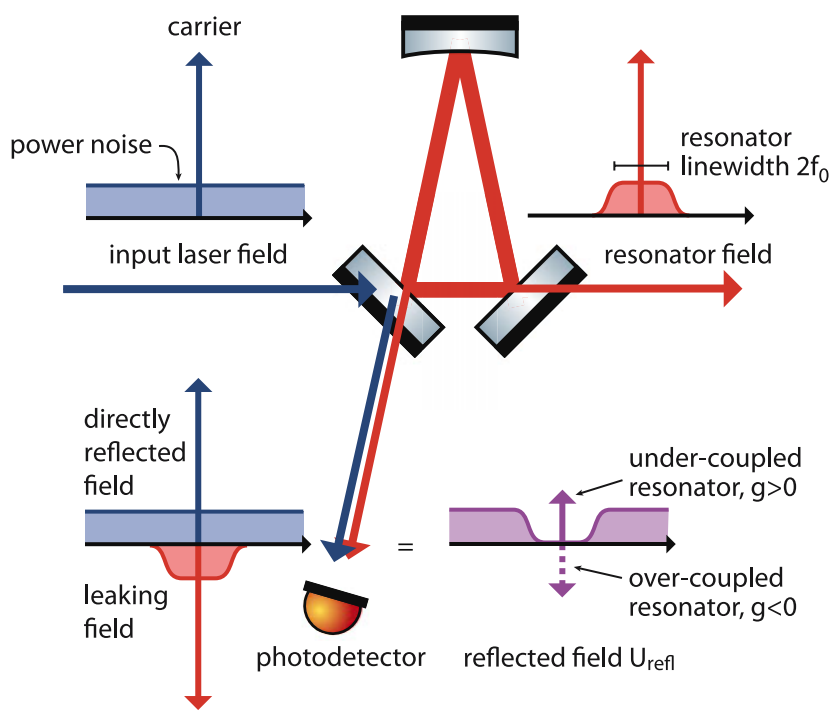

Fig. 5 Illustration of the optical fields in the sideband picture at a resonant optical resonator. The field in reflection $U_{\text {refl }}$, given by the interference of the beam directly reflected and the beam leaking from the resonator, is detected in the optical ac coupling technique

impedance matching. Conventionally, electrical ac coupling is used to measure small fluctuations on top of large dc signals or of slowly varying ones in order to increase the detection sensitivity for these fluctuations. The reflection at an optical resonator is used to create a similar effect, where the signal is attenuated at low Fourier frequencies. The optical ac coupling can be interpreted as signal conditioning in the optical domain.

The transmission of an optical resonator for power fluctuations decreases for frequencies above the resonator bandwidth [24]. Accordingly, power fluctuation sidebands are mainly reflected by the resonator at high frequencies, whereas the carrier and low-frequency sidebands are almost completely transmitted for a nearly impedance-matched resonator (Fig. 5). Thus the average power in reflection is reduced while the high frequency fluctuation sidebands are fully preserved. This effect can be described by the transfer function $G(f)$ for relative power fluctuations from the beam upstream of the resonator to the beam in reflection [23]:

$G(f)=g-(g-1) \times h(f), \quad h(f)=\frac{1}{1+\mathrm{i} f / f_{0}}$,

$|G(f)|=\sqrt{\frac{1+g^{2} \times f^{2} / f_{0}^{2}}{1+f^{2} / f_{0}^{2}}}$,

where $h(f)$ describes the approximated power fluctuation filter effect of the resonator with bandwidth $f_{0}$, and $g$ is the maximum gain for very high frequencies $\left(G\left(f \gg f_{0}\right) \rightarrow g\right)$. The parameter $g$ depends on the impedance matching of the resonator, which has to be slightly over- or under-coupled for this technique. The average power in reflection is reduced to $1 / g^{2}$, called carrier reduction, assuming a perfect

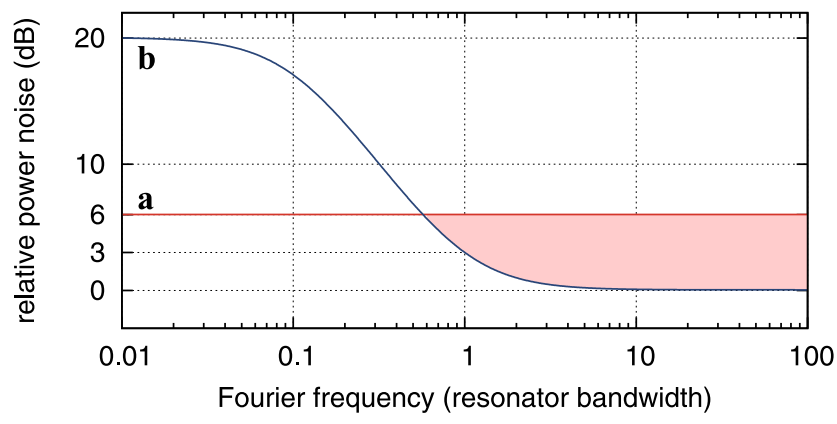

Fig. 6 Quantum-noise limits for the different power stabilization schemes, neglecting any technical limitations. The limits are given in relation to the relative quantum noise of the original beam. The optical ac coupling scheme (b) allows to go below the quantum limit of the traditional scheme (a) by up to $6 \mathrm{~dB}$ and thus to access the shaded area

mode matching. Non-resonant parasitic modes in the input beam, such as higher TEM modes or radio frequency modulation sidebands, will reduce the maximum gain $g$.

This transfer function $G(f)$ describes an optical amplification of relative power fluctuations for frequencies above the resonator bandwidth and can be used to build more sensitive power detectors. However, a special impedance matching of the resonator and a very good mode matching is required to fully exploit this effect. A gain of about $g=10$ can be realized with moderate experimental effort considering technical limitations, such as the achievable mode matching or the controllability of the impedance matching.

With this technique a new power stabilization scheme (Fig. 1b) is possible: The beam splitter and the in-loop photodetector of the traditional scheme are replaced by a compound detector, consisting of the resonator and the photodetector in reflection. This scheme not only circumvents technical limitations but also achieves a better quantum limited performance compared to the traditional scheme. A detailed analysis [21, 22] of the theoretical quantum limit of the stabilization scheme with optical ac coupling shows that for frequencies above the resonator bandwidth the quantum limit of a traditional stabilization scheme can be surpassed by $6 \mathrm{~dB}$ (Fig. 6). Associated with this better fundamental limit is the nearly doubled beam power available for downstream experiments since less power needs to be detected for the stabilization. In real experiments, however, several technical limitations have to be considered as well, such as the limited power detection capability of photodiodes. Taking these limitations into account, the stabilization scheme with optical ac coupling achieves a significantly superior performance [21, 22] at high laser power levels that will be used, e.g., in future GWDs.

\subsection{Optical ac coupling experiments}

We studied the optical ac coupling technique not only theoretically but also performed several experiments to demonstrate the feasibility of this detection scheme and its high 


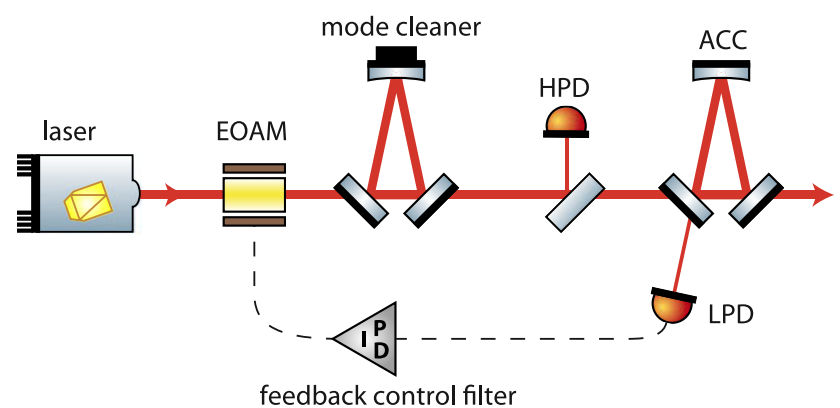

Fig. 7 Simplified experimental setup of the power sensing and power stabilization experiment with optical ac coupling. In the power sensing experiment the free-running power noise of the laser was measured with photodetector LPD. In the power stabilization experiment this detector was used as in-loop detector and the power noise was measured with the independent out-of-loop photodetector HPD. The optical resonator ACC was used for the optical ac coupling and the electro-optic amplitude modulator EOAM for actuating the laser output power. The feedback control loop was used only in the power stabilization experiment

sensitivity for power fluctuations. In one experiment, the power sensing experiment [23], the high sensitivity of an optical ac coupled photodetector was demonstrated at high frequencies of several megahertz. In another experiment, the power stabilization experiment [25], an optical ac coupled photodetector was used as the power sensor in a power stabilization control loop. This experiment demonstrated the feasibility of this new power stabilization concept at lower frequencies between $1 \mathrm{kHz}$ and $1 \mathrm{MHz}$.

The experimental setups of these two experiments are similar (Fig. 7). In both cases an NPRO with an output power of $2 \mathrm{~W}$ at a wavelength of $1064 \mathrm{~nm}$ was used as laser. The laser beam power was controlled with an EOAM for the downstream experiment. An additional optical ring resonator served as a mode cleaner in order to suppress higherorder transverse modes of the beam and to reduce beam pointing fluctuations. A mode matching of better than $99 \%$ to the subsequent resonator (ACC) used for the optical ac coupling was only possible thanks to this mode cleaner.

About $10 \%$ of the beam was sampled and detected with a photodetector (HPD) consisting of a $2 \mathrm{~mm} \mathrm{InGaAs} \mathrm{photodi-}$ ode and a low-noise transimpedance amplifier. This detector was used for an independent measurement of the laser power noise.

The remaining beam power of up to $900 \mathrm{~mW}$ was directed to the ACC. This resonator had a measured finesse of about 10,000 , a bandwidth of about $35 \mathrm{kHz}$, and was slightly under-coupled. The laser frequency was stabilized to a fundamental mode resonance of the ACC.

The beam reflected off the ACC was detected with the photodetector LPD. This photodetector consisted, just as the HPD, of an InGaAs photodiode with a low-noise transimpedance amplifier.

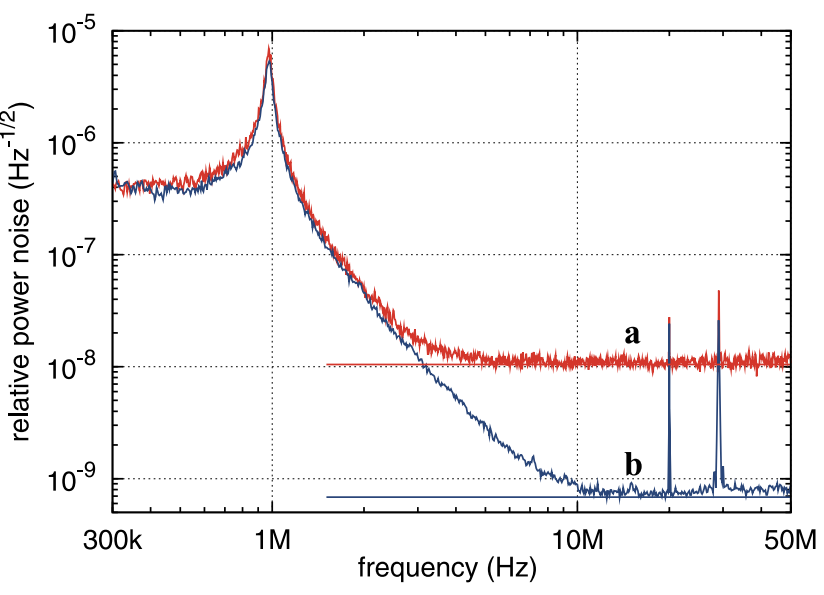

Fig. 8 Measured power noise at radio frequencies. The laser relaxation oscillation at $1 \mathrm{MHz}$ and the steep roll-off towards higher frequencies was measured. The quantum limits for the different measurements are shown as horizontal lines. The sensitivity using the optical ac coupling (b) was improved by $G(f)$, compared to the same detector with off-resonant ACC (a). The signals at $20 \mathrm{MHz}$ were injected for calibration purposes and those around $29 \mathrm{MHz}$ were due to the modulation for stabilizing the mode cleaner at a resonance

In the power sensing experiment the transfer function $G(f)$ was measured and agreed very well with the model using the parameters $f_{0}=34.4 \pm 0.3 \mathrm{kHz}$ and $g=$ $15.3 \pm 0.3$. A corresponding carrier reduction to $0.39 \%$ of the original power was measured. The relative power noise was measured once with optical ac coupling and once in a traditional setup by setting the laser frequency off-resonant to the ACC in order to verify the measurement with this new technique. In the off-resonant case the ACC acted like a high-reflective mirror and the power in front of the mode cleaner was reduced to about $0.4 \%$ to reproduce the same power level on the photodetector LPD.

The measurement of the relative power noise with optical ac coupling (Fig. 8b) agreed within the measurement and calibration accuracy $(\approx 1 \mathrm{~dB})$ up to $\approx 2 \mathrm{MHz}$ with the traditional measurement (Fig. 8a). At higher frequencies, the traditional measurement was limited by quantum noise $(1 \times$ $10^{-8} \mathrm{~Hz}^{-1 / 2}, 3 \mathrm{~mA}$ photocurrent). From about $10 \mathrm{MHz}$ the measurement with optical ac coupling was limited by quantum noise at a level of $7 \times 10^{-10} \mathrm{~Hz}^{-1 / 2}$. Even though the detector LPD was operated at a photocurrent of only $3 \mathrm{~mA} \mathrm{a}$ quantum-noise-limited sensitivity of $7 \times 10^{-10} \mathrm{~Hz}^{-1 / 2}$ was achieved, for which in a traditional setup one would have to detect a photocurrent of $702 \mathrm{~mA}$. The comparison of the two measurements with locked (Fig. 8b) and with unlocked ACC (Fig. 8a) clearly shows the gain in sensitivity of this method by $G(f)$.

In the power stabilization experiment the resonator ACC and the photodetector LPD provided a compound in-loop power sensor and the photodetector HPD was used as an outof-loop power sensor. The out-of-loop measured power sta- 


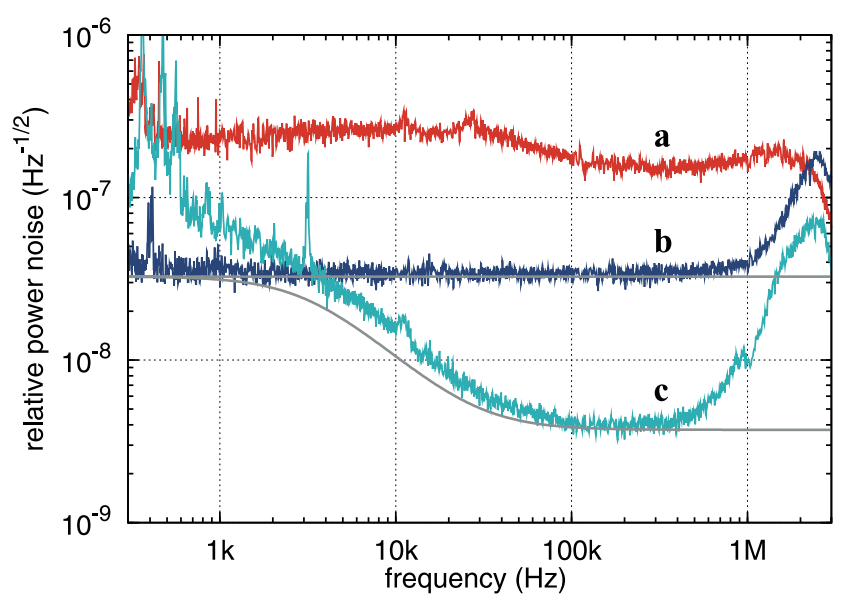

Fig. 9 Out-of-loop measured power noise at photodetector HPD using different power stabilization schemes. The free-running laser power noise (a) was reduced by the stabilization. The results are close to their quantum limits (solid lines). With optical ac coupling the power stability (c) achieved is significantly better than with the equivalent traditional stabilization scheme (b). The relaxation oscillation of the laser is not present in these measurements since it was suppressed by a laser internal pre-stabilization

bility was first limited by excess noise in the compound detector due to residual frequency noise and resonator-internal scattering. However, by improving the frequency stabilization, carefully controlling the frequency-noise coupling, and by suppressing scattered light with a resonator-internal aperture, the expected power stability was achieved.

The optical ac coupling transfer function from relative power fluctuations at detector HPD to fluctuations at detector LPD was measured again for this experiment. A fit of the model (4) to the data yielded $f_{0}=35.7 \mathrm{kHz}$ and $g=11.2$. These parameters differed from the power sensing experiment since a different ACC resonator for the optical ac coupling was used.

The out-of-loop relative power noise was measured with detector HPD in three different conditions (Fig. 9). First the free-running power noise of the laser was measured. The relative power noise was about $2 \times 10^{-7} \mathrm{~Hz}^{-1 / 2}$ which is a typical value [26] for these laser models (Fig. 9a).

Next, the power stabilization control loop was closed (Fig. 9c). The out-of-loop power noise dropped in the whole measurement frequency band by up to $33 \mathrm{~dB}$. The best power stability was $3.7 \times 10^{-9} \mathrm{~Hz}^{-1 / 2}$ at Fourier frequencies around $200 \mathrm{kHz}$. The expected limit was composed of the quantum noise of the in-loop detector (LPD, $0.3 \mathrm{~mA}$ photocurrent, relative power noise of $3.3 \times 10^{-8} \mathrm{~Hz}^{-1 / 2}$ / $G(f))$ and the quantum noise of the out-of-loop detector (HPD, $60 \mathrm{~mA}$ photocurrent, relative power noise of $2.3 \times 10^{-9} \mathrm{~Hz}^{-1 / 2}$ ). The power stability achieved was very close to this limit. For frequencies below about $1 \mathrm{kHz}$, the residual power noise was dominated by mechanical reso- nances of the setup and for frequencies above $500 \mathrm{kHz}$, the stabilization was loop gain limited.

Finally the laser frequency was tuned to be off-resonant in the ACC such that the ACC acted as a normal highreflective mirror. This setup was now equivalent to a traditional power stabilization setup. The power stabilization controller was slightly modified to compensate for the missing amplification $g$, and the beam in front of the detector LPD was attenuated. With unchanged power levels on the in-loop and out-of-loop photodetectors, the power stabilization loop was closed. As expected the power noise at the out-of-loop detector was limited at a level of the quantum noise of the in-loop detector (LPD, $0.3 \mathrm{~mA}$ photocurrent, relative power noise of $3.3 \times 10^{-8} \mathrm{~Hz}^{-1 / 2}$ ) up to Fourier frequencies of about $1 \mathrm{MHz}$ (Fig. 9b).

For Fourier frequencies above $4 \mathrm{kHz}$ the out-of-loop power stability was improved by up to a factor of 9 by using the optical ac coupling scheme.

The power sensing experiment demonstrated that the sensitivity of a photodetector can be significantly improved with the optical ac coupling technique. A sensitivity of $7 \times 10^{-10} \mathrm{~Hz}^{-1 / 2}$ was achieved, which is to our knowledge the most sensitive power noise measurement so far and in particular the first measurement below $10^{-9} \mathrm{~Hz}^{-1 / 2}$. Furthermore, the power stabilization experiment showed the feasibility of the stabilization scheme involving this advanced detection technique.

\section{Discussion}

Highly sensitive power sensors and high performance power stabilizations are very important especially for high-precision optics experiments. In the recent decade the sensitivity of these sensors and the performance of power stabilizations have been improved steadily in the field of GWDs. The highly demanding power stability requirement for the second-generation GWD Advanced LIGO has been demonstrated in a precursor laboratory experiment using a highsensitivity photodiode array. However, the maximal power handling capability of commonly used photodiodes seems to be reached.

The power handling problems can be circumvented with the optical ac coupling detection technique. This advanced detection technique was demonstrated in several laboratory table-top experiments achieving a tenfold better sensitivity for frequencies above a few $10 \mathrm{kHz}$. This frequency is determined by the bandwidth of the resonator used for the optical ac coupling such that the long resonators of GWDs will allow an application of this technique down to several hertz. The power recycling cavities of second-generation GWDs have a bandwidth of about $1 \mathrm{~Hz}$ due to their kilometer-long baseline. Therefore the sensitivity gain of the optical ac coupling technique might be used in the future to significantly 


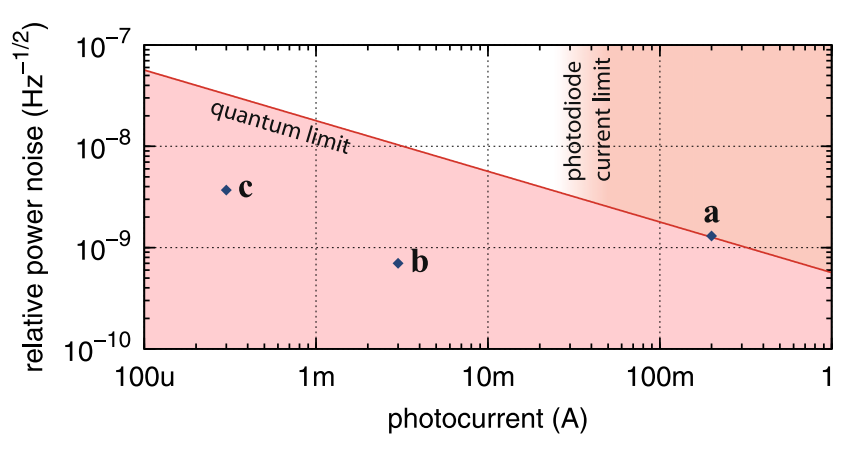

Fig. 10 Achieved sensitivity for power fluctuations in the presented experiments. The high-power photodiode array (a) pushed the photocurrent limit of a quantum-noise-limited traditional detection by scaling the number of photodiodes. The experiments with optical ac coupling, power sensing experiment (b) and power stabilization experiment (c), achieved a much higher sensitivity and surpassed the quantum limit of a traditional detection with the same average photocurrent

improve the power stability of the laser beam injected into the interferometer.

The results of the experiments described in this article are summarized in Fig. 10. The photodiode array presented pushed the photocurrent limit for a quantum-noise-limited detection at low frequencies to about $200 \mathrm{~mA}$. The optical ac coupling surpassed the fundamental quantum limit of a traditional detection by employing optical resonators and increasing the sensitivity of a photodetector without increasing the average photocurrent. Both approaches, the development of highly sensitive traditional photodetectors and the development of new advanced detection techniques, complement each other and open a whole new range of attainable power stabilities that seemed to have been inaccessible due to technical limits before.

Acknowledgements This research is supported by the DFG and is part of QUEST (Centre for Quantum Engineering and Space-Time Research). The authors would like to thank Albrecht Rüdiger for his helpful comments during the preparation of this manuscript.

\section{References}

1. S. Rowan, J. Hough, Living Rev. Relativ. 3, 1 (2000)
2. K. Somiya, Y. Chen, N. Mio, Phys. Rev. D 73, 1 (2006)

3. B. Willke, K. Danzmann, M. Frede, P. King, D. Kracht, P. Kwee, O. Puncken, R.L. Savage, B. Schulz, F. Seifert, C. Veltkamp, S. Wagner, P. Wessels, L. Winkelmann, Class. Quantum Gravity 25, 114040 (2008)

4. P. Fritschel, Proc. SPIE 4856, 282 (2003)

5. R. Flaminio, A. Freise, A. Gennai, P. Hello, P. La Penna, G. Losurdo, H. Lueck, N. Man, A. Masserot, B. Mours, M. Punturo, A. Spallicci, A. Vicere, Advanced virgo white paper. Technical Report VIR-NOT-DIR-1390-304, VIRGO Collaboration (2005)

6. K. Kuroda, the LCGT Collaboration, Class. Quantum Gravity 27, 084004 (2010)

7. H. Lück, C. Affeldt, J. Degallaix, A. Freise, H. Grote, M. Hewitson, S. Hild, J. Leong, M. Prijatelj, K. Strain, B. Willke, H. Wittel, K. Danzmann, J. Phys. Conf. Ser. 228, 012012 (2010)

8. S. Nagano, M. Musha, L. Ying, N. Mio, K. Ueda, Laser Phys. 8, 686 (1998)

9. D.J. Ottaway, P.J. Veitch, C. Hollitt, D. Mudge, M.W. Hamilton, J. Munch, Appl. Phys. B 71, 163 (2000)

10. R.S. Abbott, P.J. King, Rev. Sci. Instrum. 72, 1346 (2001)

11. I. Zawischa, M. Brendel, K. Danzmann, C. Fallnich, M. Heurs, S. Nagano, V. Quetschke, H. Welling, B. Willke, Class. Quantum Gravity 19, 1775 (2002)

12. F. Nocera, Class. Quantum Gravity 21, S481 (2004)

13. J. Rollins, D. Ottaway, M. Zucker, R. Weiss, R. Abbott, Opt. Lett. 29, 1876 (2004)

14. B.W. Barr, K.A. Strain, C.J. Killow, Class. Quantum Gravity 22, 4279 (2005)

15. F. Seifert, P. Kwee, M. Heurs, B. Willke, K. Danzmann, Opt. Lett. 31, 2000 (2006)

16. N. Mio, T. Ozeki, K. Machida, S. Moriwaki, Jpn. J. Appl. Phys. 46, 5338 (2007)

17. P. Kwee, B. Willke, K. Danzmann, Opt. Lett. 34, 2912 (2009)

18. M.S. Taubman, H. Wiseman, D.E. McClelland, H.A. Bachor, J. Opt. Soc. Am. B 12, 1792 (1995)

19. F. Seifert, Power stabilization of high power lasers for second generation gravitational wave detectors. Ph.D. thesis, Universität Hannover, 2009

20. I. Freitag, A. Tünnermann, H. Welling, Opt. Commun. 115, 511 (1995)

21. P. Kwee, Laser characterization and stabilization for precision interferometry. Ph.D. thesis, Universität Hannover, 2010

22. P. Kwee, B. Willke, K. Danzmann, J. Phys. Conf. Ser. 228, 012023 (2010)

23. P. Kwee, B. Willke, K. Danzmann, Opt. Lett. 33, 1509 (2008)

24. A.E. Siegman, Lasers, Chapter 11 (Univ. Science Books, Mill Valley 1986)

25. P. Kwee, B. Willke, K. Danzmann, Appl. Opt. 48, 5423 (2009)

26. P. Kwee, B. Willke, Appl. Opt. 47, 6022 (2008) 